\title{
Does size really matter? A comparative study on floral traits in orchids with two different pollination strategies
}

\author{
M. Lussu, A. De Agostini, A. Cogoni, M. Marignani \& P. Cortis (iD \\ Department of Life and Environmental Sciences, University of Cagliari, Cagliari, Italy
}

\section{Keywords}

Deceptive pollination; floral traits; lock and key hypothesis; pollinators; Reproductive Standardisation Index.

\section{Correspondence}

P. Cortis, Università degli Studi di Cagliari

Dipartimento di Scienze della Vita e

dell'Ambiente Viale S. Ignazio, 13 I-09123

Cagliari, Italia.

E-mail: pierluigi.cortis@unica.it

\section{Editor}

A. Dafni

Received: 19 November 2018; Accepted: 31 March 2019

doi:10.1111/plb.12993

\section{ABSTRACT}

- The lock and key hypothesis assumes that male and female genitalia match in a unique system to prevent interspecific crosses. This hypothesis is largely investigated in animals, while there is a distinct lack of studies on plants. Nevertheless, we expect the lock and key hypothesis could apply to plants with complex floral morphologies, such as orchids.

- Here we apply a comparative approach to examine the variation of floral functional traits in food- and sex-deceptive orchids. To understand if a specific deception strategy is related to a specific variation in floral traits evaluated the variation in sterile and fertile traits among species and subsequently examined the correlations between male and female reproductive organs of the same species with the aim of investigating the role of the lock and key hypothesis in deceptive orchids.

- Our results show that the functional morphology of fertile traits plays a pivotal role in limiting gene flow in species that grow in sympatry. In particular, it was observed that the Reproductive Standardisation Index (RSI) is significantly different in the two pollination strategies and that the correlation between pollinarium length and stigmatic cavity length is stronger in food-deceptive species when compared to the sex-deceptive species.

- These results reveal that the lock and key hypothesis contributes to maintain boundaries in plants with very complex floral morphology.

\section{INTRODUCTION}

The lock and key hypothesis assumes that species-specific morphological matching between male and female genitalia promotes prezygotic reproductive isolation between closely related species. The biological concept of species is based on one or more barriers that, at different levels, affect the gene flow, leading to the rise of reproductive isolation mechanisms (RIM) between populations, promoting speciation (Dobzhansky 1937; Mayr 1943; Lussu et al. 2018). In animals, the lock and key hypothesis compares the female genitalia to a lock and male genitalia to a key: the combination of the key with the correct lock leads to successful coupling (Shapiro \& Porter 1989; Coyne \& Orr 2004). What emerges is that barriers are essential for successful coupling and, in order to define a given barrier, it is necessary to fulfil two conditions: first the male and female reproductive organs must match, second the correlation between the morphology of the two sexes should be expressed (Masly 2012). This hypothesis has been investigated actively in sympatric and parapatric animal species (Schick 1965; Tanabe \& Sota 2008), and several recent investigations have shown that this mechanism contributes to retain reproductive isolation as, for instance, in the millipede genus Parafontaria (Xystodesmidae; Tanabe \& Sota 2008).

In angiosperms, a large specialisation of floral traits is often associated with a dominance of prezygotic barriers compared to postzygotic barriers, where their co-occurrence leads to the maintenance of species boundaries (Darwin 1862; Phillips et al.
2017). Although a highly specialised pollination strategy allows benefits and a reduction in pollen loss, it also has different negative side effects, such as an increasing risk of extinction (Waser 1996).

In flowering plants, studies on the role of mechanical isolation as a prezygotic barrier are often associated with ethological investigations on pollinators (Grant 1994; Jersáková et al. 2009). Grant (1994) identified two different conditions of mechanical isolation: in the first, plant species isolation is maintained by adopting different pollinators; in the second, plant species share the same pollinators but allocate pollen to different parts of a pollinator's body. The astonishing diversity of Orchidaceae is accompanied by the precise morphology of floral traits (Dressler 1993; Rudall \& Bateman 2002) that make these plants unique and immediatly recognisable. Orchid flowers are often described as having adaptive attractions to different pollinators (Cozzolino \& Widmer 2005; Vandewoestijne et al. 2008). Many authors have investigated the role of the perianth in pollinator attraction, reproductive isolation or speciation and concluded that morphological and chemical traits often contribute to limit gene flow between orchid species (Dafni 1983; Dressler 1993; Cozzolino \& Widmer 2005). Little is known, however, about the occurrence and the relevance of the lock and key hypothesis in orchids. The majority of orchids offer some kind of reward to their pollinators but nearly onethird rely on deceptive strategies (Cozzolino \& Widmer 2005; Jersáková et al. 2009). In her review on food deception, Jersáková et al. (2009) described the mechanical barrier as "the 
lock and key system whereby pollinarium adheres to orchid stigmata means that orchids can easily share pollinators with other plants without losing pollen to their stigmata."

Food-deceptive orchids are, in this context, particularly interesting because they mimic traits such as colours, scents or spurs typical of species that offer a reward (Peter \& Johnson 2006a; Scopece et al. 2009), or mimic a well-defined rewarding species (Johnson 2000; Anderson \& Johnson 2005), devising interesting mechanisms to prevent the pollinator from discovering their deception. Usually, food-deceptive orchids are generalist mimicks and are often pollinated by a variety of pollinators (Robertson 1928), then prezygotic barriers are very labile when they occur in sympatry. Pollinators are mainly naive insects, recently emerged individuals or those that explore different nectar resources (Dafni 1983; Cozzolino \& Widmer 2005).

In sex deception, plant species attract insects (especially males) by imitating shapes, colours and molecules secreted by sexually mature females (Paulus 2006). This relationship is very often species-specific, thus many orchid species are pollinated by one or a few closely related insect species (Gögler et al. 2009, 2015; Dormont et al. 2014), hence they usually display Batesian mimickry. Several studies have confirmed that sex deception strongly limits the gene flow also between closely related species, representing a strong and precise prezygotic barrier (Cortis et al. 2009; Vereecken et al. 2011; Xu et al. 2012; Whitehead \& Peakall 2014; Gögler et al. 2009, 2015; Lussu et al. 2018). The European genus Ophrys was used by Grant (1994) to name a very precise condition of the ethological mechanism.

In this study, we investigate the role of the lock and key hypothesis as RIM in orchids. In particular, we hypothesise that the sizes of the stigmatic cavity and size of the pollinarium could covary because of their ecological role in reproduction. Accordingly, their correlation is expected to be stronger in generalist orchids than in those species that adopt more specialised deception. Using a morphological and functional approach in eight sympatric orchid species as sample for the two deception strategies, we addressed the following questions: (i) do fertile and sterile traits vary within each deception strategy? Since deceptive plants prevent pollinators from associating the floral traits with the deception, we hypothesised that in food-deceptive species, sterile traits will vary more than the fertile ones because visual traits (perianth) are primarily involved in this deception strategy. (ii) Are pollinaria and the stigmatic cavity morphologies correlated - does the sexual deception show a stronger correlation; and (iii) is there a morphological correspondence between stigmatic cavity and pollinarium promoted by natural selection?

\section{MATERIAL AND METHODS}

For this study we selected eight of the most representative Mediterranean widespread and unrewarding orchid species with overlapping phenology and distribution. We have excluded from this study endemic taxa or poorly ethologically studied species ascribed to phylogenetically unresolved clades. We compiled a list of eight species, four representative of the food-deception strategy and four representative of the sexdeception strategy (for a complete list of species, see Table S1). In order to avoid individuals affected by hybridisation or introgression phenomena we have selected only samples from isolated populations. The data gathering was conducted during the blooming season of 2017 in Sardinia (Italy): food-deceptive orchids are the first to bloom, reaching a peak between March and April, in contrast sex-deceptive orchids have a tendency to bloom later, peaking between April and May. For each species, 20 plants were randomly chosen and one fresh, healthy, mature and undamaged flower from each plant was collected. Length of stigmatic cavity and pollinarium were measured in the field after removing the flower from the plant. In the absence of information about caudicle movement after removal of the pollinarium, its length was measured after $2.5 \mathrm{~min}$, the necessary time for the caudicle to bend into the ideal position (Darwin 1862; Peter \& Johnson 2006b). Pollinaria were extracted with an inoculating loop and their lengths measured from the base of the viscidium to the top of the pollinium. After removing the pollinarium, each flower was preserved in 70\% ethanol solution. In the laboratory, dissections were conducted under a stereoscopic microscope, then petals, sepals and labellum were digitised on graph paper. For each trait, length, width and area were measured using ImageJ (https://imagej.nih.gov/ij/). Fitness was calculated following the formula: $\mathrm{W}=$ no of fruits/no of flowers (Tremblay 2011).

In order to compare the intraspecific relationship between the stigmatic cavity length and the pollinarium length, we propose the Reproductive Standardisation Index (RSI) as the ratio between stigmatic cavity length and pollinarium length. To assess the difference within and between food- and sex-deceptive groups, we calculated means, SD and coefficients of variation. ANOVA was performed to assess differences in RSI between and within pollination strategy. To test the hypothesis that plant functional traits differ between the two deception strategies, we performed a permutational multivariate ANOVA (PERMANOVA; Anderson 2001) by testing the simultaneous response of all the measured traits, only the fertile traits and only the sterile traits, comparing the food- versus sex-deceptive groups. We used Euclidean distance on standardised data, 999 unrestricted permutations of raw data using correct permutable units; the pair-wise tests were corrected for multiple comparison. Pearson correlation coefficient were calculated to estimate morphological relationship between male and female traits within each strategy and the relationship between RSI and fitness. Significance was assessed with a generalised linear model with binomial distribution and logit link; fitness (W) was used as response variable and fertile traits and RI as independent variables. All the statistical analyses were performed using R Studio (R Core Team, 2017).

\section{RESULTS}

We examined 160 flowers over the duration of this study. Within sterile traits, intraspecific variation of the labellum is generally more variable than the other floral traits (Table 1). Coefficients of variation of sterile traits are 0.14 in food-deceptive strategy and 0.26 in the sexually-deceptive strategy. On the other hand, coefficients of variation of fertile traits are 0.29 and 0.12 in the food-deceptive strategy and sexually-deceptive strategy, respectively (Fig. 1). Details of each species are shown in Table S1. PERMANOVA showed that the deception strategy is significant in generating differences between plant functional traits among individuals (Total traits $F_{(1,158)}=28.4$, $P<0.001$; Sterile traits $F_{(1,158)}=35.95, P<0.001$; Fertile traits 
Table 1. Means $\pm S D$ and coefficients of variation for plant functional traits in food- and sex-deceptive strategies in orchids.

\begin{tabular}{|c|c|c|c|c|c|c|}
\hline & \multicolumn{3}{|c|}{$\begin{array}{l}\text { Food-deceptive } \\
\text { strategy }\end{array}$} & \multicolumn{3}{|c|}{$\begin{array}{l}\text { Sexually-deceptive } \\
\text { strategy }\end{array}$} \\
\hline & Mean & SD & $C V$ & Mean & SD & $\mathrm{CV}$ \\
\hline \multicolumn{7}{|l|}{ Fertile traits } \\
\hline $\begin{array}{l}\text { Stigmatic cavity } \\
\text { length }\end{array}$ & 2.93 & 0.41 & 0.15 & 2.45 & 0.46 & 0.19 \\
\hline $\begin{array}{l}\text { Stigmatic cavity } \\
\text { width }\end{array}$ & 2.54 & 0.25 & 0.10 & 2.71 & 0.81 & 0.30 \\
\hline Stigmatic cavity area & 7.68 & 1.42 & 0.20 & 6.72 & 2.63 & 0.39 \\
\hline Pollinarium length & 3.26 & 0.31 & 0.09 & 3.30 & 0.54 & 0.16 \\
\hline Mean & & & 0.14 & & & 0.26 \\
\hline RSI & 0.90 & 0.14 & 0.15 & 0.75 & 0.13 & 0.17 \\
\hline Total of fertile traits & 2.40 & 1.07 & 0.44 & 2.31 & 1.08 & 0.47 \\
\hline \multicolumn{7}{|l|}{ Sterile traits } \\
\hline Labellum height & 11.39 & 1.13 & 0.11 & 10.18 & 1.50 & 0.15 \\
\hline Labellum width & 12.60 & 1.36 & 0.11 & 11.05 & 1.85 & 0.17 \\
\hline Labellum area & 114.44 & 21.03 & 0.19 & 114.25 & 32.408 & 0.28 \\
\hline Left sepal length & 10.71 & 0.98 & 0.09 & 9.21 & 1.00 & 0.22 \\
\hline Left sepal width & 4.95 & 0.70 & 0.14 & 4.88 & 1.04 & 0.21 \\
\hline Dorsal sepal length & 9.65 & 0.95 & 0.10 & 8.42 & 1.40 & 0.17 \\
\hline Dorsal sepal width & 4.19 & 0.53 & 0.13 & 4.82 & 1.13 & 0.23 \\
\hline Left sepal length & 10.49 & 0.99 & 0.10 & 9.13 & 2.09 & 0.23 \\
\hline Left sepal width & 4.95 & 0.52 & 0.11 & 4.91 & 1.15 & 0.23 \\
\hline Right petal length & 8.64 & 0.70 & 0.08 & 5.17 & 1.62 & 0.31 \\
\hline Right petal width & 3.05 & 0.39 & 0.13 & 2.03 & 0.62 & 0.30 \\
\hline Left petal length & 8.42 & 0.92 & 0.11 & 5.22 & 1.61 & 0.31 \\
\hline Left petal width & 3.01 & 0.42 & 0.14 & 2.03 & 0.60 & 0.30 \\
\hline Mean & & & 0.12 & & & 0.24 \\
\hline Total of sterile traits & 6.85 & 3.74 & 0.54 & 5.58 & 2.84 & 0.51 \\
\hline
\end{tabular}

$\left.F_{(1,158)}=5.54, P<0.001\right)$. Considering RSI, statistically significant differences between the two deception strategies were detected $\left(F_{(1,158)}=24.8, \quad P<0.001\right)$. Generally, the results showed a significant correlation between stigmatic cavity length and pollinarium length in the same species $(r=0.546$, $P<0.001$ ), a tendency that was confirmed at the pollination strategy level (Fig. 2), but no correlation was detected between RSI and fitness $(P>0.05)$.

At the family level, multivariate analysis shows that there is evidence that RI, stigmatic cavity length and pollinarium length are significant predictors of fitness. Moreover, when decoupled, no statistical support was detected in the sex-deceptive strategy, while in the food-deceptive strategy stigmatic cavity length and pollinarium length were both found to affect fruit set.

\section{DISCUSSION}

Reproductive Isolation Mechanisms (RIM) are fundamental to the biological concept of species because they limit gene flow and maintain species boundaries (Dobzhansky 1937). To date, several RIM have been identified in plants, with Orchidaceae being one of the most emblematic families in pollination biology. In this study, we have analysed features of the intra- and interspecific variation of floral functional traits in food- and sexually-deceptive orchids, evaluating whether these matched the predictions of the lock and key hypothesis and if it is more expressed in one of the two considered strategies. The lock and key hypothesis has been largely supported in animals, especially insects, but poorly studied in plants. In the present work, several lines of evidence support the validity of this hypothesis also for orchids.

Based on the idea that flowers of deceptive Mediterranean orchids vary in different traits, such as flower colour, to prevent the association of floral traits and duplicity (Anderson 2001; Ackerman et al. 2011; Stejskal et al. 2015), we hypothesised that trait variance might differ between the two strategies. Generally, variation in sterile traits was found to be larger than variation of the fertile portions. We found that the trait with the highest variance in both strategies is the area of the labellum. In orchids, the labellum plays a key role in pollinator attraction (Bell et al. 2010) because, in both strategies, it is directly involved in plant-pollinator communication in order to avoid geitonogamy. In sexually-deceptive species the labellum is involved in scent production and its morphology is a trigger for pseudocopulation, thus even significant changes in its morphology might not be relevant as related to chemical communication with the pollinators, in fact even a small labellum is able to produce the scent necessary to attract pollinators and induce pseudocopulation. A large intra-individual variance in labellum size might play a complementary role to fragrance and colour variability (Dormont et al. 2014) in avoiding the recognition of deception and so promoting outcrossing. Indeed, in the food-deceptive strategy, adopting a high variation in colour and floral scent (Dormont et al. 2014) could avoid pollinators learning to easily recognise deception. Although several pollinators are generally recorded for these species, e.g. Anacamptis longicornu (Poir.) R. M. Bateman, Pridgeon \& M. W. Chase, stabilising selection on floral traits might be related to the most frequent and efficient species (Stebbins 1970). Different evolutionary processes, such as disruptive, fluctuating or frequency-dependent selection, have been identified in the maintenance of intraspecific variations in sterile traits (Wright 1943; Gigord et al. 2001; Turelli et al. 2001; Frey 2004; Rausher 2008). However, in both deceptive strategies, it has also been reported that this variation may also be retained through genetic mechanisms in combination with other selective agents (Rakosy et al. 2017; Xu et al. 2012). Our study was founded on comparison of the variation in sterile and fertile traits, and identification of factors that mantain intraspecific morphological variation of sterile traits might be possible through accurate manipulation experiments.

The core of the lock and key hypothesis is the size corrispondence between male and female genitalia. Our results show a positive correlation between stigmatic cavity and pollinarium length in both strategies, although this correlation was stronger in food-deceptive species. We hypothesised a size correspondence between pollinarium and stigmatic cavity in orchids due to their special plant-insect relationship and their unique flower morphology. There is no study, to our knowledge, that specifically analyses the lock and key combination in these plants. Although there was a general low variability in floral traits, our results showed a correlation between pollinarium and stigmatic cavity sizes and differences between food- and sexual-deception strategies. In plants with complex flower structures, such as orchids, mechanical isolation represents a barrier and our findings are congruent with the fact that an interaction between other mechanisms of reproductive isolation prevent hybridisation. In the most specialised species, e.g. the sexually-deceptive Ophrys, a powerful tool that retains 

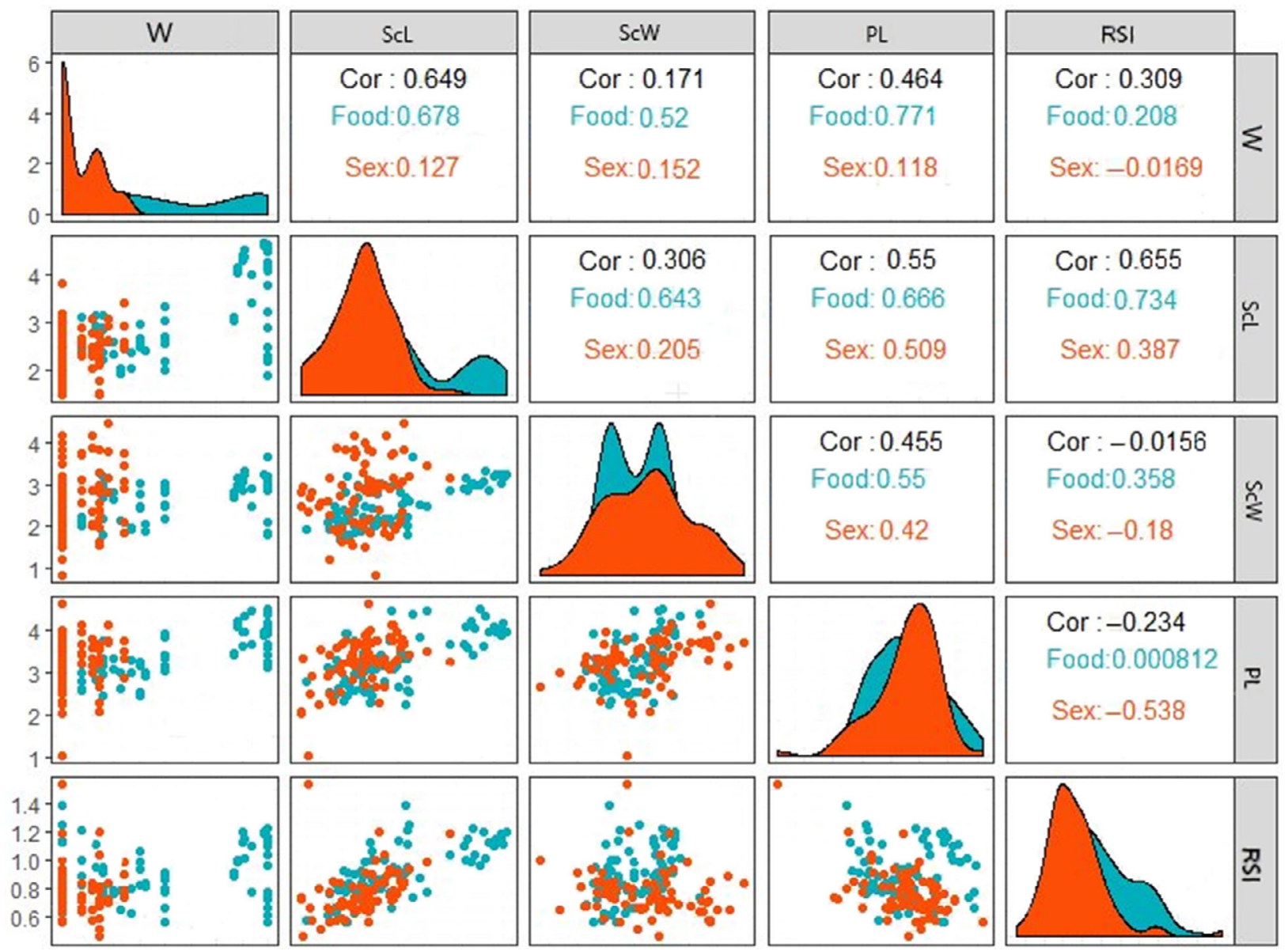

Fig. 1. Visualisation of the correlation between pairs of fertile traits, fitness and RSI. Coefficients of variation for each pair of variables are presented. Blue values are for food-deceptive strategy, orange for sexually-deceptive strategy, black values for the two strategies together. W, fitness; RSI, Reproductive Standardisation Index; CsL, stigmatic cavity length; ScW, stigmatic cavity width; PL, pollinarium length.

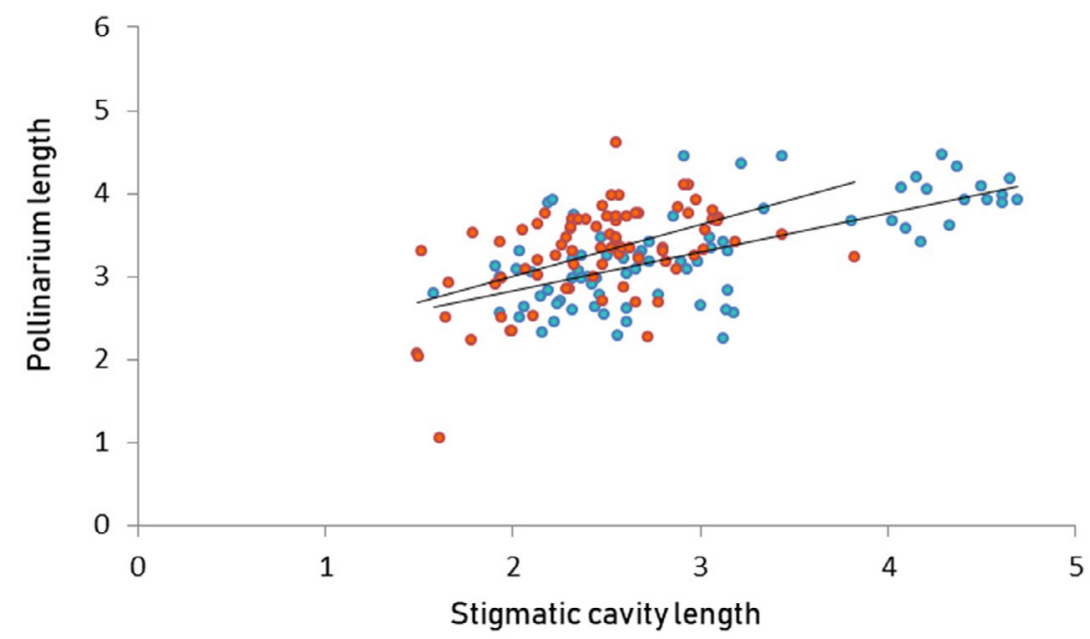

Fig. 2. Correlation among the length of the stigmatic cavity versus pollinarium length in the two deceptive strategies: in food-deceptive strategy (blue) the correlation is higher than in sexually-deceptive strategy (orange).

boundaries is the floral volatile compounds that attract one or only a few pollinators (Ayasse et al. 2000; Cozzolino \& Widmer 2005; Gögler et al. 2015). In contrast, in food-deceptive species, in which prezygotic barriers are weaker, boundaries are retained by postzygotic barriers such as different chromosome number or reduced hybrid fitness. We did not find any 
significant selection to promote the lock and key correlation. Given the potential importance of this relationship, no significant selection on these traits is surprising. Nevertheless, the fact that we did not find any statistically significant association between RSI and fruit set does not mean that a trait is not under selection. It has been reported that speciation is rapid when selection is significant; in orchids this has been identified in the diversification of the genus Ophrys in which pollinatormediated selection can drive diversification in a short period of time (Breitkopf et al. 2015; Lussu et al. 2018). We might conclude that perhaps the stigmatic cavity and pollinarium are not under strong selection if compared to those traits involved in the deception strategies. It would be interesting in the future to study this correlation over time, observing the fitness variation in different years, while also considering the effect of annual fluctuations in environmental variables.

We have demonstrated the role of morphological differences in pollinarium and stigmatic cavity in reproductive isolation in orchid species with complex floral morphologies and elaborated pollination strategies. Our results help to elucidate the importance of the lock and key hypothesis for reproductive isolation among orchids. In general, more examples of natural selection on species with complex floral traits, such as in Orchidaceae or Asclepiadaceae (Muola et al. 2011), are necessary to evaluate the role of floral morphology in retaining species boundaries. Possible future investigations should focus on comparative studies, examining hybrid fecundity, their floral compounds and seed vitality in sympatric species.

\section{SUPPORTING INFORMATION}

Additional supporting information may be found online in the Supporting Information section at the end of the article.

Table S1. Biological characteristics of species and locality of populations studied.

Table S2. Plant functional trait measures of food-deceptive species investigated.

Table S3. Plant functional trait measures of sexuallydeceptive species investigated.

\section{REFERENCES}

Ackerman J.D., Cuevas A.A., Hof D. (2011) Are deception-pollinated species more variable than those offering a reward? Plant Systematics and Evolution, 293, 91-99.

Anderson M.J. (2001) A new method for non-parametric multivariate analysis of variance. Austral Ecology, 26, 32-46.

Anderson B., Johnson S.D. (2005) The effects of floral mimics and models on each others' fitness. Proceedings of the Royal Society of London, Series B: Biological Science, 273, 969-974.

Ayasse M., Schiestl F.P., Paulus H.F., Löfstedt C., Hansson B., Ibarra F., Francke W. (2000) Evolution of reproductive strategies in the sexually deceptive orchid Ophrys sphegodes: how does flower-specific variation of odor signals influence reproductive success? Evolution, 54, 1995-2006.

Bell C.D., Soltis D.E., Soltis P.S. (2010) The age and diversification of the angiosperms re-revisited. American Journal of Botany, 97, 1296-1303.

Breitkopf H., Onstein R.E., Cafasso D., Schlüter P.M., Cozzolino S. (2015) Multiple shifts to different pollinators fuelled rapid diversification in sexually deceptive Ophrys orchids. New Phytologist, 207, 377-389.

Cortis P., Vereecken N.J., Schiestl F.P., Barone Lumaga M.R., Scrugli A., Cozzolino S. (2009) Pollinator convergence and the nature of species' boundaries in sympatric Sardinian Ophrys (Orchidaceae). Annals of Botany, 104, 497-506.

Coyne J.A., Orr H.A. (2004) Speciation. Sinauer Associates, Sunderland, MA, USA, p 545.

Cozzolino S., Widmer A. (2005) Orchid diversity: an evolutionary consequence of deception? Trends in Ecology \& Evolution, 20, 487-494.

Dafni A. (1983) Mimicry and deception in pollination. Annual Review of Ecology and Systematics, 15, 259278.

Darwin C. (1862) On the various contrivances by which British and foreign orchids are fertilised by insects, and on the good effects of intercrossing. John Murray, London, UK.

Dobzhansky T. (1937) Genetics and the orign of species. Columbia University Press, New York, USA, p 364.
Dormont L., Delle-Vedove R., Bessière J.M., Schatz B. (2014) Floral scent emitted by white and coloured morphs in orchids. Phytochemistry, 100, 51-59.

Dressler R. (1993) Phylogeny and classification of the orchid family. Cambridge University Press, Cambridge, UK, p 330.

Frey F.M. (2004) Opposing natural selection from herbivores and pathogens may maintain floral color variation in Claytonia virginica (Portulacaceae). Evolution, 58, 2426-2437.

Gigord L.D.B., Macnair M.R., Smithson A. (2001) Negative frequency-dependent selection maintains a dramatic flower color polymorphism in the rewardless orchid Dactylorhiza sambucina (L.). Proceedings of the National Academy of Sciences, USA, 98, 62536255.

Gögler J., Stökl J., Sramkova A., Twele R., Francke W. Cozzolino S., Cortis P., Scrugli A., Ayasse M. (2009) Ménage à trois - two endemic species of deceptive orchids and one pollinator. Evolution, 63, 22222234.

Gögler J., Stökl J., Cortis P., Beyrle H., Barone Lumaga M.R., Cozzolino S., Ayasse M. (2015) Increased divergence in floral morphology strongly reduces gene flow in sympatric sexually deceptive orchids with the same pollinator. Evolutionary Ecology, 25, 703-717.

Grant V. (1994) Modes and origins of mechanical and ethological isolation in angiosperms. Proceedings of the National Academy of Sciences, USA, 91, 3-10.

Jersáková J., Johnson S.D., Jürgens A. (2009) Plant-environment interactions, signalling and communication in plants, from sensory plant biology to active plant behavior. Springer, Berlin, Germany, pp 223-246.

Johnson S. (2000) Batesian mimicry in the nonrewarding orchid Disa pulchra, and its consequences for pollinator behaviour. Biological Journal of the Linnean Society, 71, 119-132.

Lussu M., De Agostini A., Marignani M., Cogoni A., Cortis P. (2018) Ophrys annae and Ophrys chestermanii: an impossible love between two orchid sister species. Nordic Journal of Botany, 2018, e01798.

Masly J.P. (2012) 170 years of lock and key: genital morphology and reproductive isolation. International Journal of Evolutionary Biology, 2012, 1-10. Article ID 247352.
Mayr E. (1943) Criteria of subspecies, species and genera in ornithology. Annals of the New York Academy of Sciences, 44, 133-139.

Muola A., Mutikainen P., Laukkanen L., Lilley M., Leimu R. (2011) The role of inbreeding and outbreeding in herbivore resistance and tolerance in Vincetoxicum hirundinaria. Annals of Botany, 108, 547-555.

Paulus H.F. (2006) Deceived males - pollination biology of the Mediterranean orchid genus Ophrys (Orchidaceae). Journal Europäischer Orchideen, 38, 303-351.

Peter C.I., Johnson S.D. (2006a) Anther cap retention prevents self-pollination by elaterid beetles in the South African orchid Eulophia foliosa. Annals of Botany, 97, 345-355.

Peter C.I., Johnson S.D. (2006b) Doing the twist: a test of Darwin's cross-pollination hypothesis for pollinarium reconfiguration. Biology Letters, 2, 65-68.

Phillips R.D., Brown G.R., Dixon K.W., Hayes C., Linde C.C., Peakall R. (2017) Evolutionary relationships among pollinators and repeated pollinator sharing in sexually deceptive orchids. Journal of Evolutionary Biology, 30, 1674-1691.

R Core Team (2017) R: a language and environment for statistical computing. R Foundation for Statistical Computing, Vienna, Austria. Available from https:// www.R-project.org/.

Rakosy D., Cuervo M., Paulus H.F., Ayasse M. (2017) Looks matter: changes in flower form affect pollination effectiveness in a sexually deceptive orchid. Journal of Evolutionary Biology, 30, 1978-1993.

Rausher M.D. (2008) Evolutionary transitions in floral color. International Journal of Plant Science, 169, 721.

Robertson C. (1928) Flowers and insects; List of visitors of four hundred and fifty-three flowers. University of Illinois, Carlinville, IL, USA, p 221.

Rudall P.J., Bateman R.M. (2002) Roles of synorganisation, zygomorphy and heterotopy in floral evolution: the gynostemium and labellum of orchids and other lilioid monocots. Biological Reviews of the Cambridge Philosophical Society, 77, 403-441.

Schick R.X. (1965) The crab spiders of California (Araneida, Thomisidae). Bulletin of the American Museum of Natural History, 129, 1-184. 
Scopece G., Musacchio A., Widmer A., Cozzolino S. (2009) Contrasting thoughts about deceptive orchids: a response to Sobel and Randle. Evolution, 63, 2205-2209.

Shapiro A.M., Porter A.H. (1989) The lock-and-key hypothesis: evolutionary and biosystematic interpretation of insect genitalia. Annual Review of Entomology, 34, 231-245.

Stebbins G.L. (1970) Adaptive radiation of reproductive characteristics in angiosperms, I: pollination mechanisms. Annual Review of Ecology, Evolution, and Systematics, 1, 307-326.

Stejskal K., Streinzer M., Dyer A., Paulus H.F., Spaethe J. (2015) Functional significance of labellum pattern variation in a sexually deceptive orchid (Ophrys heldreichii): evidence of individual signature learning effects. PLoS ONE, 10, e0142971.
Tanabe T., Sota T. (2008) Complex copulatory behavior and the proximate effect of genital and body size differences on mechanical reproductive isolation in the millipede genus Parafontaria. The American Naturalist, 171, 692-699.

Tremblay R.L. (2011) Fitness landscapes in orchids: parametric and non-parametric approaches. Lankesteriana, 11, 355-362.

Turelli M., Schemske D.W., Bierzychudek P. (2001) Stable two-allele polymorphisms maintained by fluctuating fitnesses and seed banks: protecting the blues in Linanthus parryae. Evolution, 55, 1283-1298.

Vandewoestijne S., Schtickzelle N., Baguette M. (2008) Positive correlation between genetic diversity and fitness in a large, well-connected metapopulation. BMC Biology, 6, 46 .
Vereecken N.J., Streinzer M., Ayasse M., Spaethe J., Paulus H.F., Stökl J., Cortis P., Schiestl F.P. (2011) Integrating past and present studies on Ophrys pollination - a reply to Bradshaw et al.. Botanical Journal of the Linnean Society, 165, 329-335.

Waser N.M. (1996) Disintegration of the ecological community. The American Naturalist, 104, 501-528.

Whitehead M.R., Peakall R. (2014) Pollinator specificity drives strong prepollination reproductive isolation in sympatric sexually deceptive orchids. Evolution, 68, 1561-1575.

Wright S. (1943) An analysis of local variability of flower color in Linanthus parryae. Genetics, 28, 139-156.

Xu S., Schlüter P.M., Schiestl F.P. (2012) Pollinatordriven speciation in sexually deceptive orchids. International Journal of Ecology, 2012. Article ID 285081. https://doi.org/10.1155/2012/285081 